\title{
Article \\ Kalman Filter Implementation of Subglottal Impedance-Based Inverse Filtering to Estimate Glottal Airflow during Phonation
}

\author{
Juan P. Cortés ${ }^{1,+*}$, Gabriel A. Alzamendi ${ }^{2}$, Alejandro J. Weinstein ${ }^{3}$, Juan I. Yuz ${ }^{1}$, Víctor M. Espinoza ${ }^{4}$, Daryush D. \\ Mehta ${ }^{5,6,7}$, Robert E. Hillman ${ }^{5,6,7}$, and Matías Zañartu ${ }^{1}$ \\ 1 Department of Electronic Engineering, Universidad Técnica Federico Santa María, Valparaíso, Chile \\ 2 Institute for Research and Development on Bioengineering and Bioinformatics, CONICET-UNER, Oro Verde, \\ Entre Ríos, Argentina \\ 3 Department of Biomedical Engineering, Universidad de Valparaíso, Chile \\ 4 Department of Sound, Universidad de Chile, Chile \\ Massachusetts General Hospital, Boston, MA \\ Harvard Medical School, Boston, MA \\ MGH Institute of Health Professions, Boston, MA \\ * Correspondence: juan.cortess@usm.cl \\ + Current address: Affiliation 1
}

check for

updates

Citation: Cortés, J.P.; Alzamendi,

G.A.; Weinstein, A.J.; Yuz, J.I.;

Espinoza, V.M.; Mehta, D.D.; Hillman,

R.E.; Zañartu, M. Title. Preprints 2021,

1, 0. https://doi.org/

Received:

Accepted:

Published:

Publisher's Note: MDPI stays neutral with regard to jurisdictional claims in published maps and institutional affiliations.

\begin{abstract}
Subglottal Impedance-Based Inverse Filtering (IBIF) allows for the continuous, non-invasive estimation of glottal airflow from a surface accelerometer placed over the anterior neck skin below the larynx, which has been shown to be advantageous for the ambulatory monitoring of vocal function. However, during long-term ambulatory recordings over several days, conditions may drift from the laboratory environment where the IBIF parameters were initially estimated due to sensor positioning, skin attachment, and temperature, among other factors. Observation uncertainties and model mismatch may result in significant deviations in the glottal airflow estimates, but are very difficult to quantify in ambulatory conditions due to a lack of a reference signal. To address this issue, we propose a Kalman filter implementation of the IBIF filter, which allows for both estimating the model uncertainty and adapting the airflow estimates to correct for signal deviations. One-way ANOVA results from laboratory experiments using the Rainbow Passage indicate a an improvement on amplitude-based measures for PVH subjects compared to IBIF which shows a statistically difference with respect to the reference oral airflow $(p=0.02, F=4.1)$. MFDR from PVH subjects is slightly different to the oral airflow when compared to $\operatorname{IBIF}(p=0.04, F=3.3)$. Other measures did not have significant differences with either Kalman or IBIF, with the exception of H1H2, whose performance deteriorates for both methods. Overall, both methods show similar flottal airflow measures, with the advantage of Kalman by improving amplitude estimation. Moreover, Kalman filter deviations from the IBIF output airflow might suggest a better representation of some fine details in the ground-truth glottal airflow signal. Other applications may take more advantage from the adaptation offered by the Kalman filter implementation.
\end{abstract}

Keywords: Vocal Hyperfunction; Inverse Filtering; Kalman Filter

\section{Introduction}

Voice disorders are a health problem of significant concern in our society. In the United States, voice disorders affect about $7 \%$ of the working population [1-4]. Many of these voice disorders are chronic or recurring conditions that result from repeated detrimental patterns of vocal behavior, referred to as vocal hyperfunction $(\mathrm{VH})[5,6]$. Subtypes of $\mathrm{VH}$ include phonotraumatic $\mathrm{VH}(\mathrm{PVH})$ that is associated with the formation of benign vocal fold (VF) lesions (e.g., nodules) due to phonotrauma and non-phonotraumatic VH that is associated with the dis-coordination of laryngeal muscle control in the absence of structural abnormalities (often diagnosed as primary muscle tension dysphonia) [7]. In spite of the significant prevalence of these disorders, very little is known about the underlying physical mechanisms of $\mathrm{VH}$. Given that multiple factors contribute and interact in different ways to cause and sustain $\mathrm{VH}$ disorders, there are non-specific, broad-based behavioral treatments 
that are inefficient, make patient compliance more challenging, and make it difficult or impossible to link improvements in vocal function to specific parts of the therapy program [8].

Several efforts have been carried out to develop objective measures that can capture $\mathrm{VH}$, such as aerodynamic measures obtained from estimates of the glottal airflow $[5,9,10]$, relative fundamental frequency [11], estimates of spectral tilt of the voice source [12,13], and cepstral-related measures [14], among others. However, these measures are typically applied in the context of a laboratory assessment with sustained vowels and do not capture the nuances of $\mathrm{VH}$ in natural speech during daily activities.

The objective assessment of $\mathrm{VH}$ is expected to be significantly enhanced through ambulatory monitoring of vocal function. Ambulatory voice monitoring aims at providing complementary information that current clinical methods cannot offer. An ambulatory approach that could precisely pinpoint the instance, duration, and type of $\mathrm{VH}$ behavior would have the capability to provide transformative advancements in how clinical practices monitor, evaluate, and treat VH. Efforts in ambulatory methods are heading in this direction [15-17], but there are many associated challenges.

Some of the ambulatory voice monitors use either a microphone signal to estimate fundamental frequency and jitter [18], a surface electromyograph to estimate increased muscle tension [19], or a neck-surface accelerometer over the extrathoracic trachea to estimate sound pressure level, fundamental frequency, voicing activity, vocal dose, and related measures[20-26], as well as aerodynamic, cepstral and related parameters [14,27]. Aerodynamic measures have been successfully used to differentiate both phonotraumatic and non-phonotraumatic VH patients from matched controls using sustained vowels $[9,28]$, and have been shown to become salient features of compensatory mechanisms associated with VH in modeling studies [29,30]. Thus, these aerodynamic measures have a strong potential to enhance the ability to identify VH in ambulatory settings [27].

Given that traditional assessment of aerodynamic signals using a Rothenberg mask [31] is not feasible for ambulatory scenarios, indirect estimation methods are required. The Subglottal Impedance-Based Inverse Filtering (IBIF) scheme [32] allows for estimating the glottal airflow signal from neck-surface vibration. The IBIF approach was recently tested in a discrimination task using week-long ambulatory recordings for 50 patients with vocal fold nodules and 50 matched healthy-control subjects [27]. The results of classification task using aerodynamic features outperformed previous efforts with other measures $[14,15,17,24]$ and provides a new avenue to improve the assessment and treatment of VH disorders.

In spite of these advances, unquantified uncertainties are associated to the estimation of the glottal airflow signal with the IBIF scheme due to a number of factors. First, the determination of the IBIF model parameters uses inverse filtering of the oral airflow from few sustained vowel samples, which can lead to IBIF parameter variations for different vowels and pitch conditions [27,33]. The latter becomes especially challenging for highpitched female voices, which are common in ambulatory studies. In addition, there are combined measurement uncertainties from the accelerometer due to sensor positioning, skin attachment, temperature, etc. Furthermore, there is no direct reference that can be used to quantify these combined effects in ambulatory scenarios. Thus, there is a need to quantify the magnitude of the uncertainty in the estimation process, and to potentially improve the estimation of the aerodynamic signals through the IBIF framework.

To address the aforementioned limitations, we propose a Kalman filter (KF) implementation of the IBIF filter, which allows for both estimating the uncertainty and adapting the airflow estimates to correct for potential deviations in the airflow signal estimates. The KF structure is a standard Kalman filter with a forward pass in the signal. However, due to the time-dependent structure of the states (i.e., the glottal airflow signal), delayed states are estimated based on information of future observations, which is a smoothed estimation of the signal calculated in real time by the KF. To assess the accuracy of the KF, we compare measures estimated from the glottal airflow signal obtained from the oral mask using a 
notch-filter [34] and from IBIF [32] with a group of PVH and healthy-controls subjects reading a phonetically balanced passage.

The paper is structured as follows: In section 2, we present the methods utilized to estimate glottal airflow, namely the IBIF method and its Kalman filter implementation. Then, in section 3, we describe the experimental setup with participants with $\mathrm{PVH}$ and vocally healthy control subjects. In section 4 , we present the results of the experiments, and in section 5, we discuss them in detail. Finally, in section 6, we present the conclusions and future work.

\section{Materials and Methods}

\subsection{Standard IBIF Implementation}

The IBIF scheme is described in the frequency domain, where the glottal airflow (also referred to as glottal volume velocity, GVV) and the acceleration signal are related by

$$
\dot{U}_{\text {skin }}(\omega)=T_{\text {skin }}(\omega) \cdot U_{\text {sub }}(\omega)
$$

where $\dot{U}_{\text {skin }}(\omega)$ is the acceleration signal, $U_{\text {sub }}(\omega)$ is the inverted GVV (assuming source is a dipole, that is, two equal and opposite volume velocity sources [35]), and $T_{\text {skin }}(\omega)$ is the neck-skin transfer function. In what follows, we removed the frequency dependency $\omega$ in the expressions for the sake of clarity. $T_{\text {skin }}$ can be modeled by:

$$
T_{\text {skin }}=\frac{\dot{U}_{\text {skin }}}{U_{\text {sub }}}=\frac{H_{\text {sub } 1} \cdot Z_{\text {sub } 2} \cdot H_{d}}{Z_{\text {sub } 2}+Z_{\text {skin }}},
$$

where $H_{\text {sub } 1}$ is the frequency response of the subglottal section from the glottis to the accelerometer location, and $H_{d}=j \omega$ is a derivative filter (similar to the lip radiation effect, except that in this case is the acceleration in free field). $Z_{\text {sub } 2}$ is a frequency-dependent driving-point impedance corresponding to the subglottal section [36] below the accelerometer position. $Z_{\text {skin }}$ is the neck-skin impedance modeled as a mechanical analog of an RLC circuit in series:

$$
Z_{\text {skin }} \cdot A_{a c c}=R_{m}+j\left(\omega M_{m}-\frac{K_{m}}{\omega}\right)+\frac{j \omega M_{a c c}}{A_{a c c}},
$$

where $R_{m}, M_{m}$, and $K_{m}$ are the per-unit-area resistance, inertance, and stiffness of the skin, respectively. The radiation impedance due to the accelerometer loading is modeled as a derivative term $j \omega$ times the mass $M_{a c c}$ divided by the surface $A_{a c c}$ (per-unit-area) of the accelerometer and the coating or mounting disk attached to it [37]. These parameters are subject specific, and therefore involve calibration factors that can be fitted per subject using a reference GVV signal and an optimization method. The calibration factors $Q=\left\{Q_{i}\right\}_{i=1, \ldots, 5}$ are defined as:

$$
\begin{aligned}
Q & =\left\{Q_{1}, Q_{2}, Q_{3}, Q_{4}, Q_{5}\right\} \\
R_{m} & =2320 \cdot Q_{1} \\
M_{m} & =2.4 \cdot Q_{2} \\
K_{m} & =491000 \cdot Q_{3} \\
L_{\text {trachea }} & =10 \cdot Q_{4} \\
L_{\text {sub } 1} & =5 \cdot Q_{5}
\end{aligned}
$$

$$
\begin{array}{r}
{\left[\mathrm{g} \cdot \mathrm{s}^{-1} \cdot \mathrm{cm}^{-2}\right],} \\
{\left[\mathrm{g} \cdot \mathrm{cm}^{-2}\right],} \\
{\left[\mathrm{dyn} \cdot \mathrm{cm}^{-3}\right],} \\
{[\mathrm{cm}],} \\
{[\mathrm{cm}] .}
\end{array}
$$

Given the $Q$ factors above, the impulse response of neck-skin $h(n)$ is obtained by first taking the fast Fourier transform (FFT) of $T_{\text {skin }}(\omega)$ with $N$ points, which becomes $T_{\text {skin }}(k)$ with $k=0,1, \ldots, N-1$, where $N$ is the number of FFT points. Then, by forcing $T_{\text {skin }}(k)$ 
to be conjugate symmetric $\left(T_{\text {skin }}(k)=T_{\text {skin }}^{*}(N-k)\right)$, we take the inverse FFT to obtain a real impulse response $h(n)$. In this way, the resulting IBIF filter is a deterministic FIR filter of length $N$. One limitation of this approach is the assumption of fixed $Q$ factors for each subject. However, these factors contain certain degree of uncertainty $[33,38]$ due to small changes either in the mechanical properties of the neck-skin tissue, as well as changes in the effective length of the trachea when the speaker is voicing in continuous speech. Therefore, a better approach to estimate the GVV signal would be to consider the uncertainty associated to the estimation process with an adaptive filter.

\subsection{Formulation of IBIF Model Based on a Kalman Filter}

Even though the IBIF algorithm performs well in laboratory settings where the calibration procedure is done with a Rothenberg mask, there are uncertainties related to the application of the IBIF filter in ambulatory settings. First, the position and arrangement of the sensor during in field monitoring might not match laboratory specifications, so the subject-specific parameters could change slightly. One approach for tracking relevant latent signals (i.e., GVV) of a given process (i.e., IBIF) based on related noisy/perturbed observations (i.e., neck-skin acceleration) is the use of a Bayesian approach, which allows to simultaneously estimate both the unknown signal and its uncertainty [39]. Under the assumption of linearity and Gaussian distributions for the posterior distributions of the unknown states, a Kalman Filter is the optimal estimator. In this work, we propose an alternative formulation of IBIF combining the state-space framework with the moving average (MA) canonical form [40] obtained from the $h(n)$ impulse response:

$$
\begin{array}{r}
\mathbf{x}(n+1)=\mathbf{A} \mathbf{x}(n)+\mathbf{w}(n), \\
y(n)=\mathbf{C} \mathbf{x}(n)+v(n) .
\end{array}
$$

What follows is the instantiation of a Kalman filter from the model (10) and (11), to our particular problem, where $x(n)$ is the state vector that will contain the GVV signal: $x(n)=\left[\begin{array}{lllll}x(n-N+1) & x(n-N+2) & x(n-N+3) & \cdots & x(n)\end{array}\right]^{T}$ where $N$ is the length of the skin-impulse response. This, the transition matrix $\mathbf{A}$ is given by:

$$
\mathbf{A}=\left[\begin{array}{cccccc}
0 & 1 & 0 & \ldots & 0 & 0 \\
0 & 0 & 1 & \ldots & 0 & 0 \\
\vdots & \vdots & \ddots & \vdots & \vdots & \vdots \\
0 & 0 & 0 & \ldots & 0 & 1 \\
0 & 0 & 0 & \ldots & 0 & 0
\end{array}\right] \in \mathbb{R}^{N \times N}
$$

and $\mathbf{w}(n)$ is a Gaussian process noise with zero mean and covariance matrix:

$$
\mathbf{R}_{\mathbf{w}}=\left[\begin{array}{ccccc}
0 & 0 & \ldots & 0 & 0 \\
0 & 0 & \ldots & 0 & 0 \\
\vdots & \vdots & \ddots & \vdots & \vdots \\
0 & 0 & \ldots & 0 & 0 \\
0 & 0 & \ldots & 0 & \sigma_{w}^{2}
\end{array}\right] \in \mathbb{R}^{N \times N}
$$

The initial condition is specified with the mean $\mathbf{m}_{0}=\mathbf{E}\left(\mathbf{x}_{0}\right)$ and covariance $\mathbf{P}_{0}=\mathbf{E}\left(\left(\mathbf{x}_{0}-\right.\right.$ $\left.\left.\mathbf{m}_{0}\right)\left(\mathbf{x}_{0}-\mathbf{m}_{0}\right)^{T}\right)$ of the initial state $\mathbf{x}_{0}$.

The observation equation (11) relates the accelerometer signal $y(n)$ as the convolution between the unobserved state $\mathbf{x}$ and the neck-skin impulse response $h(n)$ with coefficients: 


$$
\mathbf{C}=\left[\begin{array}{lllll}
h(0) & h(1) & h(2) & \cdots & h(N-1)
\end{array}\right]^{T} \in \mathbb{R}^{1 \times N}
$$

According to (11), Gaussian measurement noise $v(n)$ with mean zero and variance $\sigma_{v}^{2}$ is assumed to perturb the observed signal. Implementation of the standard MA Kalman filter with a finite discrete-time set $\mathrm{n}=1, \ldots, \mathrm{T}$ is described in Algorithm 1 :

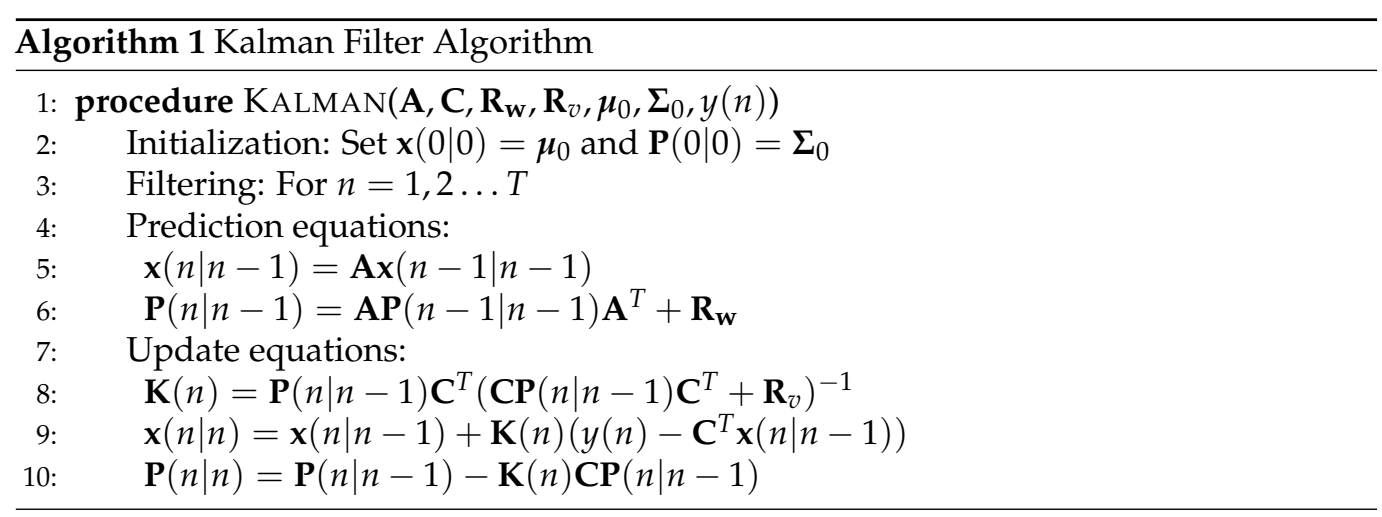

\subsection{Glottal flow model as input to Kalman filter}

According to Fant's source-filter theory of speech production [41], the glottal excitation is assumed independent of the vocal tract. Even though there is evidence for certain cases of non-linear coupling between the glottal source and the vocal tract [22,42], the source-filter theory has served well for the development of glottal source modeling and estimation. In terms of modeling the glottal source, parametric time domain models, have been proposed the Rosenberg model of glottal pulse [43] and the Lijecrants-Fant (LF) model of the derivative of the glottal pulse [44] are widely used parametric models in the time domain, that serve as template for deriving other source modeling strategies [45].

\subsubsection{Glottal source as a low-pass filter}

The source-filter theory describes the glottal source as a low-pass system in the spectral domain. Therefore, the glottal flow is the response of this low-pass filter to an impulse train excitation. Originally, Fant [41] used four poles on the negative real axis to model the glottal source:

$$
U_{g}(s)=\frac{U_{g 0}}{\prod_{i=1}^{4}\left(1-\frac{s}{s_{r i}}\right)},
$$

where $\left|s_{r 1}\right| \simeq\left|s_{r 2}\right|=2 \pi 100 \mathrm{~Hz}$ and $s_{r 3}=2 \pi 2000 \mathrm{~Hz}, s_{r 4}=2 \pi 4000 \mathrm{~Hz}$, and $U_{g 0}$ is a gain factor. According to Fant, $s_{r 3}$ and $s_{r 4}$ are fixed, whereas $\left|s_{r 1}\right|$ and $\left|s_{r 2}\right|$ account for variability to regard speaker and voice stress, where the fundamental frequency $f_{0}$ is included. Therefore, this is a 6 parameters spectral model $\left(f_{0}, U_{g 0}\right.$, and the four poles) [46].

This glottal flow model has been used in several applications, including derivation of linear prediction equations for speech [47], where only two poles are used due to the linearity of the acoustic model that only holds for frequencies below $4000 \mathrm{~Hz}$. In this case, the model simplifies to three parameters: the constant gain $U_{g 0}, f_{0}$ and a frequency parameter $s_{r 1} \simeq s_{r 2}$. Therefore, the spectral characteristics of the glottal pulse follow a second-order filter frequency response, with a spectral tilt of about $-12 d B /$ oct.

A second-order low-pass Butterworth filter [48] has been applied to model the glottal source. The magnitude-squared frequency response of a second-order Butterworth filter is: 


$$
|H(\Omega)|^{2}=\frac{G_{0}^{2}}{1+\left(\Omega / \Omega_{c}\right)^{4}}
$$

where $\Omega_{c}$ is the cut-off frequency approximately at $-3 d B$ and $G_{0}$ is the DC gain. In the $s$ domain $(s=\sigma+j \Omega)$, Eq. (13) can be rewritten as:

$$
H(s) H(-s)=\frac{G_{0}^{2}}{1+\left(-s^{2} / \Omega_{c}^{2}\right)^{2}} .
$$

The poles $s_{k}$ of the $H(s)$ filter correspond to:

$$
s_{k}=\Omega_{c} e^{j \pi / 2} e^{j(2 k+1) \pi / 4}, \quad k=0,1 .
$$

The transfer function corresponds to:

$$
H(s)=\frac{\Omega_{c} G_{0}}{\left(s-s_{0}\right)\left(s-s_{1}\right)} .
$$

The impulse invariance method $[48,49]$ is used to convert the filter to its digital form, so the mapping:

$$
z=e^{s T}
$$

is used, where $T=1 / f_{s}$ is the sampling period and $f_{s}$ the sampling frequency. Therefore, the poles corresponding to the $z$ domain are $z_{0}=e^{s_{0} T}$ and $z_{1}=e^{s_{1} T}$. By substituting the poles Eq. (15) into the transfer function (16), we obtain the following:

$$
H(z)=\frac{\Omega_{\mathcal{c}}^{2}}{\left(z-e^{-\frac{\sqrt{2}}{2} \Omega_{c}(1+j)}\right)\left(z-e^{-\frac{\sqrt{2}}{2} \Omega_{c}(1-j)}\right)} .
$$

By expanding and doing some algebraic work, the following transfer function is obtained:

$$
H(z)=\frac{\Omega_{c}^{2} z^{-2}}{1-2 \alpha_{1} z^{-1}+\alpha_{2} z^{-2}},
$$

where $\alpha_{1}=e^{\frac{-\sqrt{2}}{2} \Omega_{c} T} \cos \frac{\sqrt{2}}{2} \Omega_{c} T, \alpha_{2}=e^{-\sqrt{2} \Omega_{c} T}$, and $\Omega_{c}=2 \pi f_{c}$, where $f_{c}$ is the cut-off frequency of the low-pass filter $H(z)$.

Using Eq. (19) we can modify matrix A from the general state-space framework (see Eq. (10)) to an AR model, so the white noise entering the system is "colored" by the low-pass filter representing the glottal source. The new $\mathbf{A}_{\mathbf{l p}}$ matrix is:

$$
\mathbf{A}_{\mathbf{l p}}=\left[\begin{array}{cccccc}
0 & 1 & 0 & \ldots & 0 & 0 \\
0 & 0 & 1 & \ldots & 0 & 0 \\
\vdots & \vdots & \ddots & \vdots & \vdots & \vdots \\
0 & 0 & 0 & \ldots & 0 & 1 \\
0 & 0 & 0 & \ldots & -2 \alpha_{1} & \alpha_{2}
\end{array}\right] \in \mathbb{R}^{N \times N}
$$

An example of the estimated GVV using matrix $\mathbf{A}_{\mathbf{l p}}$ is shown in Fig. 1 and compared to the estimated GVV using the original matrix $\mathbf{A}$. The upper plot shows the tracking of the first state, which corresponds to the smoothed (time delayed estimate) GVV. It is visible that there are no differences between the original MA Kalman filter and the one 
incorporating a low-pass filter as input noise. There is, however, a noticeable difference in the tracking of the last state of the GVV, which corresponds to the the filtered GVV estimate considering all the past samples up to the current sample $n$. The original Kalman produces a zero-mean signal, while the Kalman with a low-pass input tracks a very-small amplitude signal similar to an expected GVV.

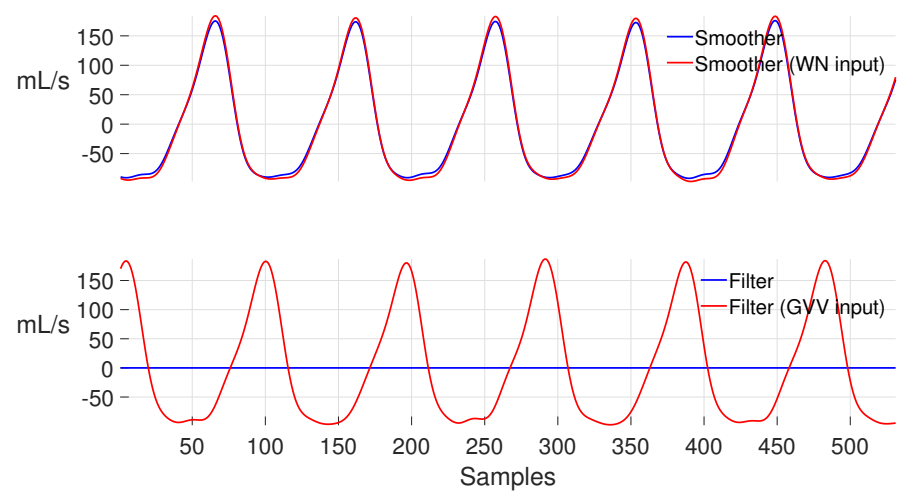

Figure 1. Top panel: GVV estimates (first state) using A (blue) and $\mathbf{A}_{\mathbf{l p}}$ (red). Bottom panel: GVV estimates (last state) using $\mathbf{A}$ (blue) and $\mathbf{A}_{\mathbf{l p}}$ (red).

\subsubsection{Rosenberg model for the glottal pulse}

A parametric model of the glottal pulse can be obtained from the Rosenberg model [43], which can be formulated as [50]:

$$
g[n]= \begin{cases}0.5\left[1-\cos (\pi(n+1)) / N_{1}\right], & 0 \leq n \leq N_{1}-1 \\ \cos \left(0.5 \pi\left(n+1-N_{1}\right) / N_{2}\right), & N_{1} \leq n \leq N_{1}+N_{2}-2 \\ 0, & \text { otherwise }\end{cases}
$$

where $N_{1}$ is the number of samples of the opening phase and $N_{2}$ is the number of samples of the closing phase. For a sequence of 96 samples (equivalent to approx. $210 \mathrm{~Hz}$ fundamental frequency and a pitch period of $4.8 \mathrm{msec}$.), with $N_{1}=25$ and $N_{2}=10$, the $z$-transform $G(z)$ has the form:

$$
G(z)=z^{-33} \prod_{k=1}^{33}\left(-b_{k}^{-1}\right) \prod_{k=1}^{33}\left(1-b_{k} z\right)
$$

where $b_{k}$ corresponds to the zeros of $G(z)$, which can also be written in the following form:

$$
\begin{aligned}
G(z) & =g[0]+g[1] z^{-1}+g[2] z^{-2}+\cdots+g[N-1] z^{-(N-1)}, \\
& =\beta_{0}+\beta_{1} z^{-1}+\beta_{2} z^{-2}+\cdots+\beta_{N-1} z^{-(N-1)} \\
& =\sum_{k=0}^{N-1} \beta_{k} z^{-k}
\end{aligned}
$$

The glottal pulse time-domain waveform $g[n]$ and its spectrum are plotted in Fig. 2

The periodic comb excitation $p[n]$ is modeled as one-sided quasi-periodic impulse train:

$$
p[n]=\sum_{k=0}^{\infty} \gamma^{k} \delta\left[n-k N_{p}\right]
$$

which has z-transform: 

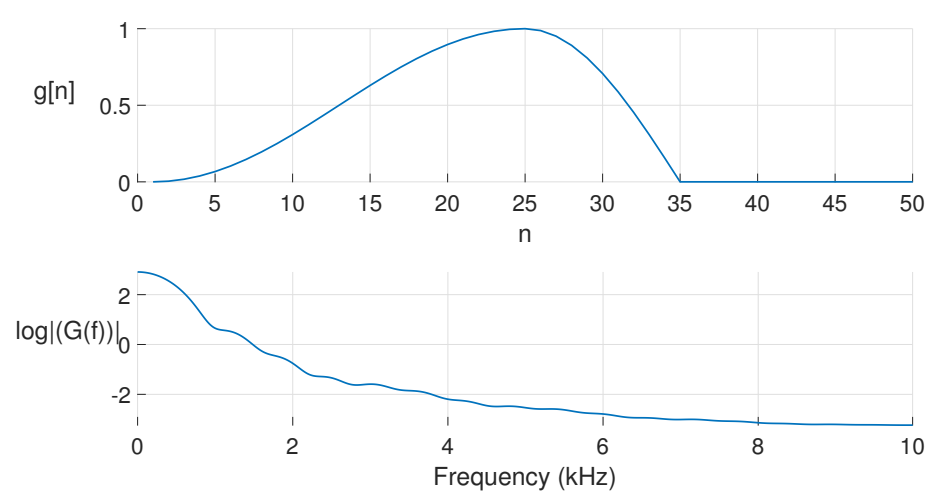

Figure 2. Rosenberg model in time domain (only first 50 samples shown, top panel) and the magnitude of its spectrum (bottom panel).

$$
P(z)=\sum_{k=0}^{\infty} \gamma^{k} z^{-k N_{p}}=\frac{1}{1-\gamma z^{-N_{p}}},
$$

where $N_{p}=f_{s} / f_{0}$ (fundamental period in samples) and $\gamma$ is a number close to 1 (e.g, $0.999)$ to make the filter stable. The spectrum of the periodic input $P(z)$ has a fundamental frequency of $f_{0}=210 \mathrm{~Hz}\left(N_{p}=96\right)$.

Therefore, $P(z) G(z)$ is the $z$-transform of the glottal flow model (spectrum shown in Fig 3). In the time-domain, the model is an ARMA model that can be constructed as a shaping filter $(s f)$ input to the canonical MA model (see Eq. (24)) [40,51]:

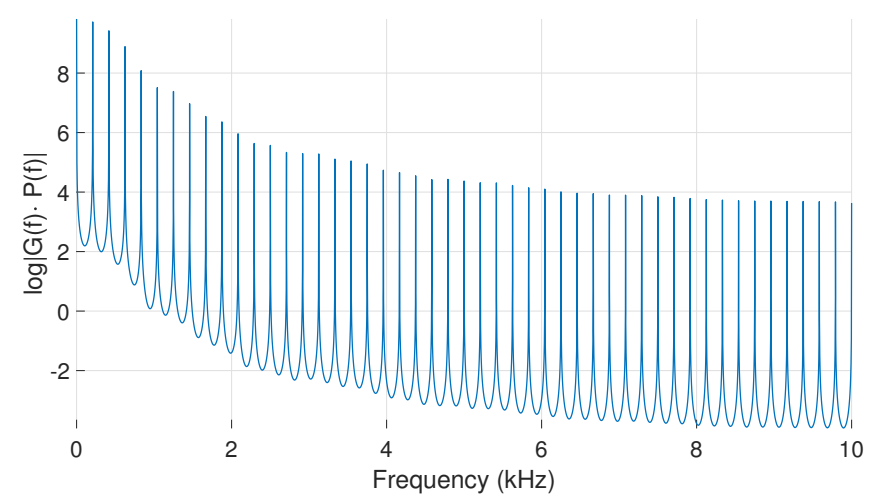

Figure 3. Spectrum of periodic input $\mathrm{P}(\mathrm{z})$ multiplied in the frequency domain by Rosenberg model $\mathrm{G}(\mathrm{z})$, which corresponds to an ARMA model of the glottal source $\left(f_{0}=210 \mathrm{~Hz}\right)$.

$$
x_{s f}(n)=-\sum_{k=1}^{p} \alpha_{k} x_{s f}(n-k)+\sum_{k=0}^{q} \beta_{k} w_{2}(n-k),
$$

where $x_{s f}(n)$ is the state of the shaping filter, $\alpha_{k}=-\gamma_{k}$ and $\beta_{k}$ are the $k^{t h}$ coefficient of the AR and MA model, respectively, and $w_{2}(n)$ is Gaussian noise with mean 0 and variance $\sigma_{w_{2}}^{2}$. The state-space equation for this model is:

$$
\begin{aligned}
\mathbf{x}_{\mathbf{S F}}(n+1) & =\mathbf{A}_{\mathbf{S F}} \mathbf{x}_{\mathbf{S F}}(n)+\mathbf{B}_{S F} w_{2}(n) \\
w_{1}(n) & =\mathbf{C}_{\mathbf{S F}} \mathbf{x}_{\mathbf{S F}}(n)
\end{aligned}
$$

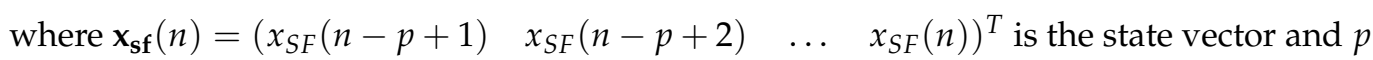
is the order of the AR model. Since the periodic input has $N_{p}$ poles, the order of the AR 
model is $p=N_{p}$. A $\mathbf{S F}$ is the transition matrix $p \times p$ :

$$
\mathbf{A}_{\mathbf{S F}}=\left[\begin{array}{cccccc}
0 & 1 & 0 & \ldots & 0 & 0 \\
0 & 0 & 1 & \ldots & 0 & 0 \\
\vdots & \vdots & \ddots & \vdots & \vdots & \vdots \\
0 & 0 & 0 & \ldots & 0 & 1 \\
-\alpha_{p} & -\alpha_{p-1} & -\alpha_{p-2} & \ldots & -\alpha_{2} & -\alpha_{1}
\end{array}\right] \in \mathbb{R}^{p \times p}
$$

$\mathbf{B}_{S F}=\left[\begin{array}{ll}0 & 0 \ldots 1\end{array}\right]^{T} \in \mathbb{R}^{p \times 1}$ and $w_{2}(n)$ is a stochastic driving noise with zero mean and variance $\sigma_{w_{2}}^{2}$. The MA equation Eq. (26) contains $\mathbf{C}_{\mathbf{S F}}=\left[\begin{array}{llll}\beta_{q} & \beta_{q-2} \ldots \beta_{1} & \beta_{0}\end{array}\right] \in \mathbb{R}^{1 \times(q+1)}$ and the colored noise $w_{1}(n) \in \mathbb{R}$ is the dot product of $\mathbf{C}_{\mathbf{S F}}$ and $\mathbf{x}_{\mathbf{S F}}(n)$. The colored noise $w_{1}(n)$ is the input to the MA state model in Eq. (27). A continuous time diagram of this augmented system is shown in Fig 4. The white noise $w_{2}(t)$ is the input to the shaping filter, which is the Rosenberg model convolved with the periodic input (Fig 3). The output of this filter is the colored white noise $w_{1}(t)$, which is the input noise to the canonical MA system (physical system in Fig 4), whose output $z(t)$ is the observed signal, i.e., the neck-skin acceleration. The new state-space equations in discrete-time are:

$$
\begin{array}{r}
\mathbf{X}_{T}(n+1)=\mathbf{A}_{T} \mathbf{X}_{T}(n)+\mathbf{B}_{T} w_{2} \\
z(n)=\mathbf{C}_{T} \mathbf{X}_{T}(n)+v(n)
\end{array}
$$

where

$$
\begin{gathered}
\mathbf{A}_{T}=\left[\begin{array}{ll}
\mathbf{A} & \mathbf{C}_{S F} \\
\mathbf{0} & \mathbf{A}_{S F}
\end{array}\right] \\
\mathbf{B}_{T}=\left[\begin{array}{c}
\mathbf{0} \\
\mathbf{B}_{S F}
\end{array}\right] \\
\mathbf{C}_{T}=\left[\begin{array}{ll}
\mathbf{C} & \mathbf{0}
\end{array}\right] \\
\mathbf{X}_{T}=\left[\begin{array}{c}
\mathbf{x} \\
\mathbf{x}_{S F}
\end{array}\right] .
\end{gathered}
$$

The proposed implementation of the IBIF method in a Kalman filter framework has two important additions: The adaptive tracking of the GVV signal using the accelerometer and the modeling of state and observation noise. In the first case, the adaptive tracking is performed through the sample by sample correction of the expected accelerometer signal by the Kalman gain $\mathbf{K}(n)$. In our hypothesis, the correction aids to improve the estimation of the GVV signal by minimizing the deviations from the GVV signal obtained with IBIF. The process noise variance $\sigma_{w}^{2}\left(\mathrm{ml}^{2} / \mathrm{s}^{2}\right)$ and the observation noise variance $\mathbf{R}_{v}=\sigma_{v}^{2}\left(\mathrm{~cm}^{2} / \mathrm{s}^{4}\right)$ were selected using a grid-search process in which we compare the root-mean-square error (RMSE, $m l / s)$ between the Kalman state $x(n-N+1)$ and a reference GVV signal obtained by inverse filtering an oral volume velocity (OVV) signal [9]. Figure 5 shows different values of $\sigma_{w}^{2}$ and $\sigma_{v}^{2}$ where multiple minima $(R M S E=17.268)$ are found within a range for one subject saying the vowel /a/. Most blue RMSE values in Figure 5 corresponds to $R M S E=17.273$ which are very close to the minimum. Similar trends were found for other subjects and vowels. We selected $\sigma_{w}^{2}=100$ and $\sigma_{v}^{2}=1$ in this work, which are plausible values for the state and measurement noises due to the assumption of higher process noise 


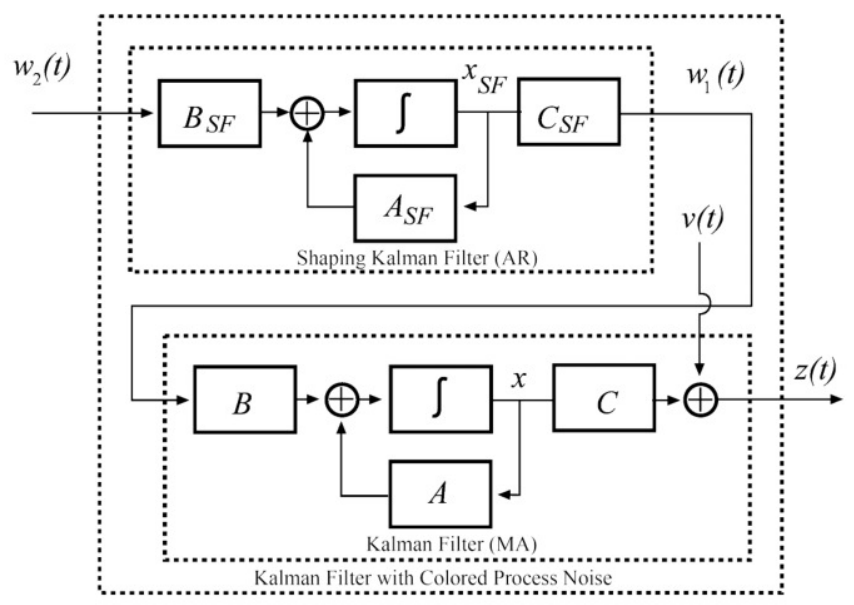

Figure 4. Diagram of augmented Kalman Filter with colored process input noise in the continuous time domain. The physical system corresponds to the standard moving average (MA) Kalman Filter, with a shaping Kalman filter based on a autoregressive (AR) noise process from the spectrum of a periodic Rosenberg glottal flow excitation.

due to glottal flow variance with low observation noise, while they produce a minimum RMSE value.

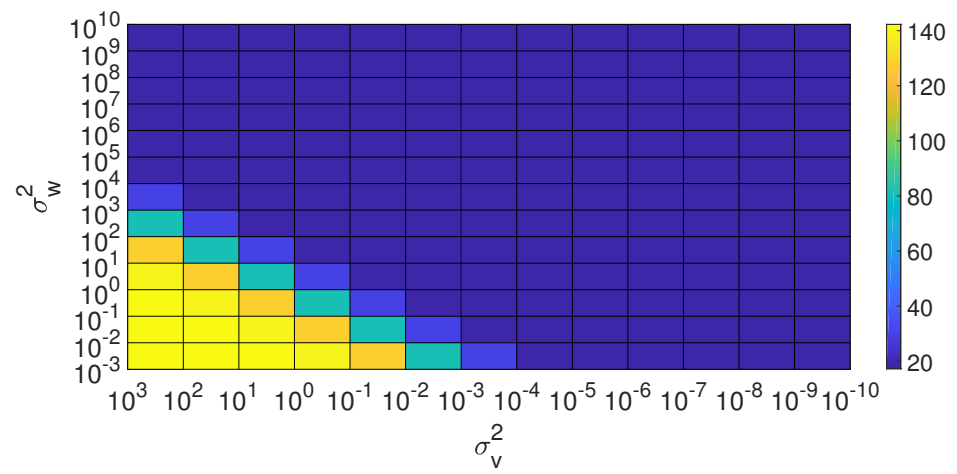

Figure 5. RMSE values for different combinations of $\sigma_{w}^{2}$ and $\sigma_{v}^{2}$.

\section{Experimental Setup}

The human studies protocol used to collect the data for this study was approved by the Institutional Review of the Mass General Brigham (formerly, Partners Healthcare System) at the Massachusetts General Hospital. Study participants were 50 pairs of adult females (total of 100 subjects) with each pair comprised of one patient with PVH (diagnosed with vocal fold nodules) and one normal control subject matched to the patient by age and occupation. Due to the higher incidence of female patients with PVH than male in the overall population [52,53] and potential sex-specific effects (e.g., due to differences in fundamental frequency), only females were selected for this study. The patient matching was done to normalize for general vocal behavior differences. Clinical diagnoses were based on a complete team evaluation by laryngologists and speech-language pathologists at the Massachusetts General Hospital Voice Center that included (a) a complete case history, (b) endoscopic imaging of the larynx, (c) aerodynamic and acoustic assessment of vocal function [54], (d) a patient-reported Voice-Related Quality of Life questionnaire [55], and (e) a clinician-administered Consensus Auditory-Perceptual Evaluation of Voice 
assessment (CAPE-V) [56]. All patients were enrolled prior to the administration of any voice treatment. Written informed consent was obtained from all subjects. The average (standard deviation) age of all subjects was 25.0 (10.5) years old.

Each subject was recorded reading a phonetically balanced text (Rainbow Passage, [57]), at a comfortable loudness level, using a Voice Health Monitor (VHM) system that consists of an accelerometer attached to the front of the neck below the larynx and connected to an smartphone application [14]. Also, synchronized recordings of oral airflow volume velocity provided a reference signal from which glottal airflow could be extracted using standard inverse filtering [31]. The sampling frequency for each signal is $20,000 \mathrm{~Hz}$ with an average of 30 seconds per passage. A typical set-up of the accelerometer attached to the neck surface is shown in Figure 6.

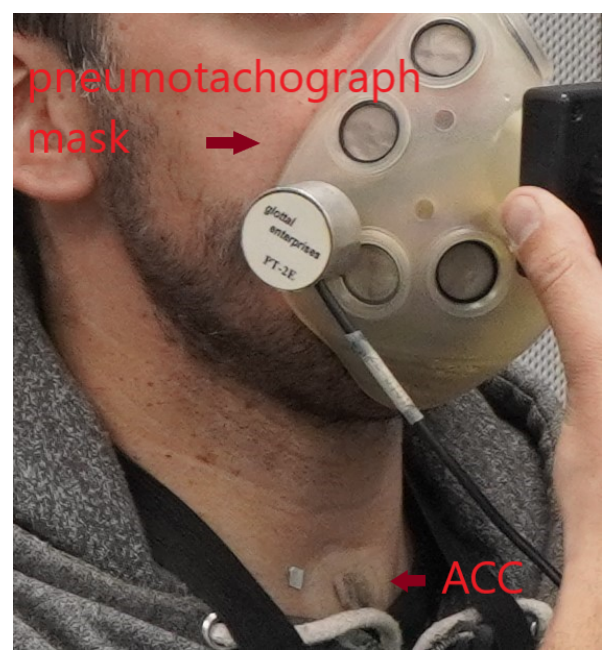

Figure 6. Experimental setup with oral airflow (pneumotachograph) mask and accelerometer sensor (ACC) on neck-surface location.

\subsection{IBIF calibration}

Each subject underwent a session in the laboratory to obtain a subject-specific calibration for the IBIF algorithm. The session involved simultaneous and synchronous recordings of a circumferentially vented mask-based oral airflow volume velocity (OVV) and necksurface acceleration in an acoustically treated room. Each subject performed a series of sustained vowel gestures (/a/ and / $\mathrm{i} /$ ) with a constant pitch using comfortable and loud (approximately $6 \mathrm{~dB}$ increase) voice. For each gesture, a bandpass-filtered $(60-1100 \mathrm{~Hz})$ oral airflow vowel segment was used to perform inverse filtering with a single notch filter (SNF) constrained to unitary gain at DC [34].

Once a glottal airflow approximation was obtained from the OVV signal, the previously introduced $\mathbf{Q}$ parameters were estimated using the optimization scheme described in [32]. These are the parameters describing the mechanical properties of the neck skin, as well as the length of the trachea and the position of the accelerometer with respect to the glottis [32].

\subsection{Ground truth GVV}

A ground truth GVV signal is necessary to compare the performance of the proposed algorithm. However, a measurement of GVV is infeasible because there is no sensor available to directly measure the airflow in the glottis. An alternative is to obtain a GVV estimate from an external sensor, e.g., an oral flow mask. Following the same method for IBIF calibration (Section 3.1), the SNF method is used in this work to calculate the ground truth GVV, which has been successfully applied in previous work related to GVV 
estimation in sustained vowels $[5,9,28,58]$. Since we have running speech in this case, the optimization procedure that finds the best notch frequency and bandwidth is done in every $50 \mathrm{~ms}$ non-overlapping frame. A simple voice activity detector based on the autocorrelation method [50] is used to remove unvoiced frames. The signal is reconstructed from individual frames by using the overlap-and-add method [50].

\subsection{Reducing order of the IBIF filter}

In order to reduce the complexity of the Kalman filter, we need to reduce the size of the matrices $\mathbf{A}$ and $\mathbf{C}$ in Eq. (10) and Eq. (11). This is necessary due to the computational cost of Kalman filter in the multiplications of state-space matrices of size $550 \times 550$ when processing running speech. Since $\mathbf{A}$ and $\mathbf{C}$ depend on the length of the neck-skin impulse response $h(n)$, the latter is truncated in the middle region and then windowed (Hann function) to 350 points. This procedure seeks to maintain the performance of IBIF filter because most of the energy of the impulse response is concentrated in the middle section, while the extremes are considerably low in energy. As an example, Figure 7 shows a given $h(n)$ in blue and the resulting truncated version in red. The magnitude of the frequency response is shown in Figure 8.

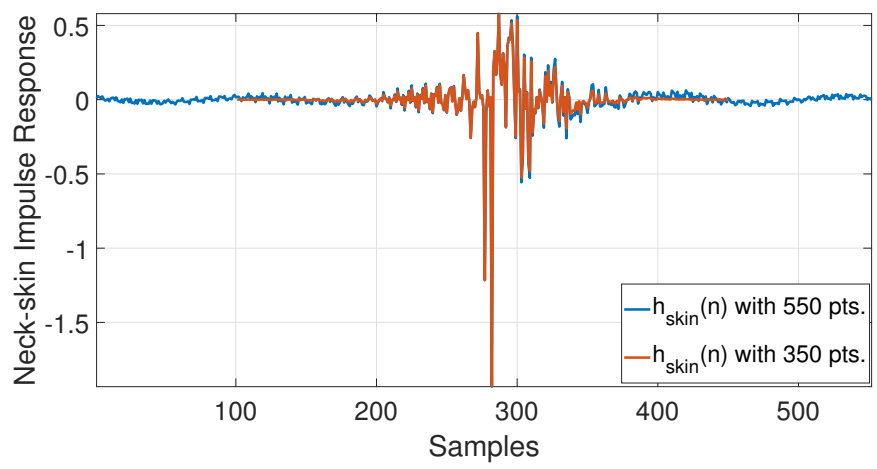

Figure 7. Neck-skin impulse response for a healthy female subject, full impulse (blue) and truncated version with a Hanning window (red).

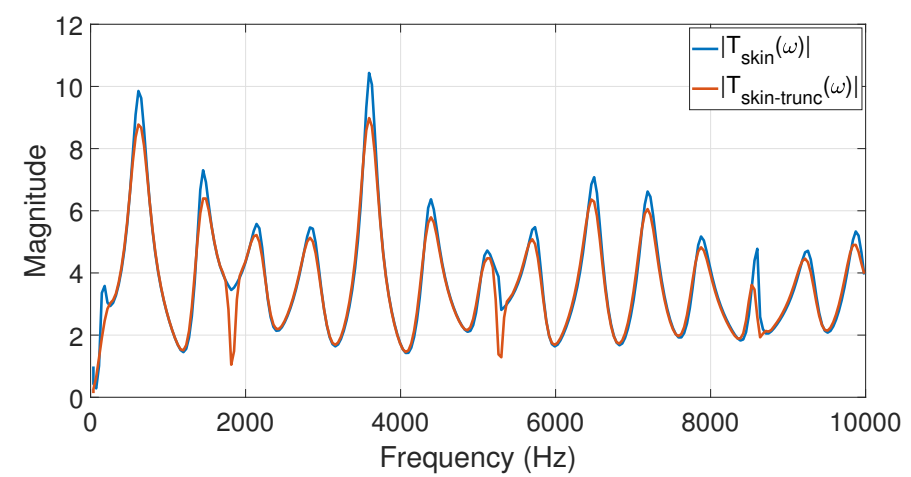

Figure 8. Neck-skin frequency response for a healthy female subject, full length (blue) and truncated version with a Hann window (red).

\subsection{Aerodynamic features}

The GVV signals from IBIF, SNF, and Kalman methods are divided in 50 ms, nonoverlapping, frames. Voicing is detected by calculating the normalized autocorrelation of the ACC signal and the main peak exceeds a threshold of 0.8 . If the frame is voiced, measures are extracted from the GVV waveform, its time-derivative, and spectrum. Figure 9 (A) shows an ACC frame and (B) a GVV waveform, the spectrum (C) and the timederivative waveform (D). The features used for this work are described in Table 1. Some of 
these aerodynamic features, such as AC flow (ACFL) and maximum flow declination rate (MFDR), have been shown to be useful to discriminate between subjects with PVH and healthy controls $[5,9,27,28,58]$. Instead of estimating time-domain features based on the detection of glottal opening and/or closing instants, the normalized amplitude quotient (NAQ) is calculated in this study, due to its robustness to noisy measurements and its correlation to the close quotient of the glottal cycle [59]. For time-domain measures (ACFL, MFDR, NAQ and $f_{0}$ ), the median for all cycles within the $50 \mathrm{~ms}$ frame is obtained. The difference in magnitude of the first and second harmonic (H1-H2) is computed from the GVV spectrum.

(a)

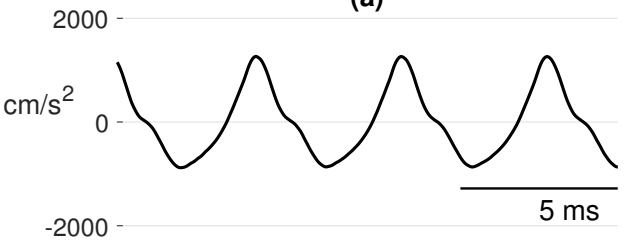

(c)

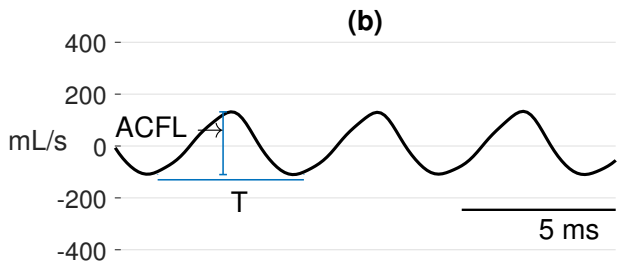

(d)

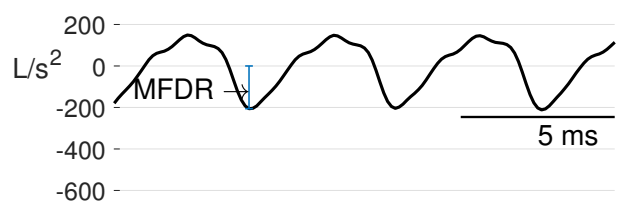

$-600$

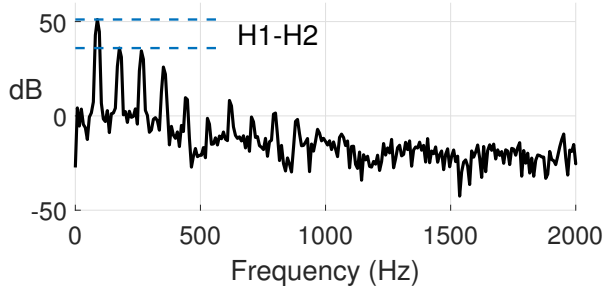

Figure 9. (a) ACC frame, (b) GVV frame, (c) spectrum from (b), and (d) time-derivative from (b).

\section{Results}

Table 2 shows summary statistics (mean \pm standard deviation) of average values, per subject, of ACFL, MFDR, H1-H2, NAQ, and $f_{0}$ from the Rainbow passage speech data, across PVH and healthy subjects, calculated with SNF, IBIF, and KF implementation of IBIF with colored noise input. Mean values are not statistically different for across the three methods similar across the three methods. The mean values of ACFL and MFDR from healthy subjects are not significant different (one-way ANOVA: $F=1.8, p=0.2$ for ACFL, $F=2.7, p=0.07$ for MFDR), while significant different between the SNF and IBIF methods for ACFL from PVH subjects $(F=4.1, p=0.02)$ and the SNF and Kalman methods for MFDR from the same group $(F=3.3, p=0.04)$. Figure 10 shows box plots for some of the measures. Overall, the distribution of measures are similar when using IBIF and the Kalman filter algorithm. Using a Brown-Forsythe test for equality of variances [60], the variance of means are not significantly different from the SNF method for ACFL (normals: $p=0.07, F=2.7$, PVH: $p=0.09, F=2.4$ ) and MFDR (PVH: $p=0.1, F=2.4)$. The variance of $\mathrm{H} 1-\mathrm{H} 2$ and NAQ means are significantly different $(p<0.05)$ when the three methods are compared.

We can observe some differences between the IBIF and KF methods when estimating the peak-to-peak amplitude (e.g., ACFL). Figure 11 shows a voiced segment of the Rainbow passage from a vocally healthy female. The SNF method (blue line) estimates a reasonably good fit of the GVV waveform from the SNF method ( $R M S E=24.9 \mathrm{ml} / \mathrm{s}$ ). However, the IBIF method does not follow as well the same ground truth signal ( $R M S E=42.7 \mathrm{ml} / \mathrm{s})$. The peak-to-peak amplitude is smaller and the close phase contains a large resonance. However, the KF method improves the estimation of the peak-to-peak amplitude for the same segment. There is some phase distortion in the closed and opening phase, but overall, the waveform has a closer match to the SNF method than IBIF. The errors to IBIF could be attributed to the production of vowels whose spectra are substantially different to a steady 


\begin{tabular}{llc}
\hline Glottal airflow measures & Description & Units \\
\hline ACFL & Peak-to-peak glottal airflow. & $\mathrm{mL} / \mathrm{s}$ \\
MFDR & Negative peak of the first derivative of the glottal waveform. & $\mathrm{L} / \mathrm{s}^{2}$ \\
H1H2 & Difference between the magnitude of the first two harmonics. & $\mathrm{dB}$ \\
Normalized Amplitude Quotient (NAQ) & Ratio of ACFL to MFDR divided by the glottal period. & - \\
Fundamental frequency $\left(f_{0}\right)$ & Inverse of the glottal period. & $\mathrm{Hz}$ \\
\hline
\end{tabular}

Table 1: Frame-based derived glottal airflow measures.

\begin{tabular}{lcccccc} 
& & ACFL & MFDR & H1-H2 & NAQ & $f_{0}$ \\
\hline \multirow{3}{*}{ PVH } & SNF & $238.8 \pm 74.9$ & $279.5 \pm 102.0$ & $11.1 \pm 1.30$ & $0.19 \pm 0.02$ & $202.4 \pm 20.1$ \\
& IBIF & $306.8 \pm 147.7$ & $346.8 \pm 178.6$ & $10.3 \pm 4.89$ & $0.20 \pm 0.05$ & $202.6 \pm 20.3$ \\
& Kalman & $287.6 \pm 131.3$ & $357.3 \pm 199.1$ & $9.69 \pm 5.51$ & $0.19 \pm 0.05$ & $200.8 \pm 20.1$ \\
\hline \multirow{3}{*}{ Healthy } & SNF & $184.5 \pm 47.0$ & $199.7 \pm 77.8$ & $10.7 \pm 1.49$ & $0.19 \pm 0.02$ & $204.6 \pm 20.9$ \\
& IBIF & $212.2 \pm 82.9$ & $260.0 \pm 122.1$ & $8.73 \pm 3.63$ & $0.19 \pm 0.04$ & $204.6 \pm 21.0$ \\
& Kalman & $199.7 \pm 77.8$ & $266.1 \pm 138.3$ & $7.73 \pm 3.85$ & $0.18 \pm 0.04$ & $203.5 \pm 21.7$ \\
\hline
\end{tabular}

Table 2: Mean and \pm standard deviation from a pool of average values of ACFL, MFDR, H1-H2, NAQ, and $f_{0}$ extracted from the Rainbow Passage (voiced frames only).

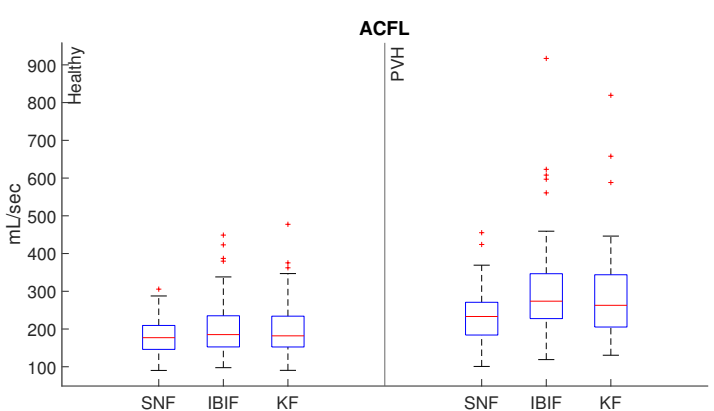

(a)

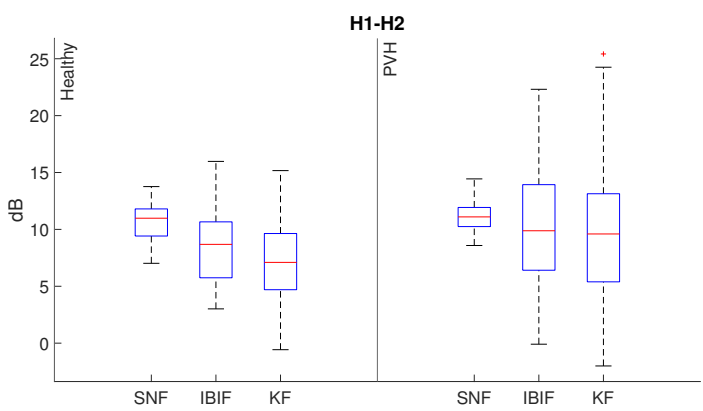

(c)

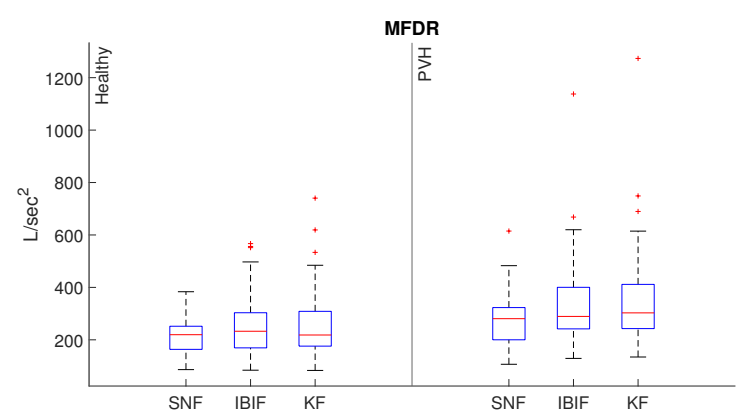

(b)

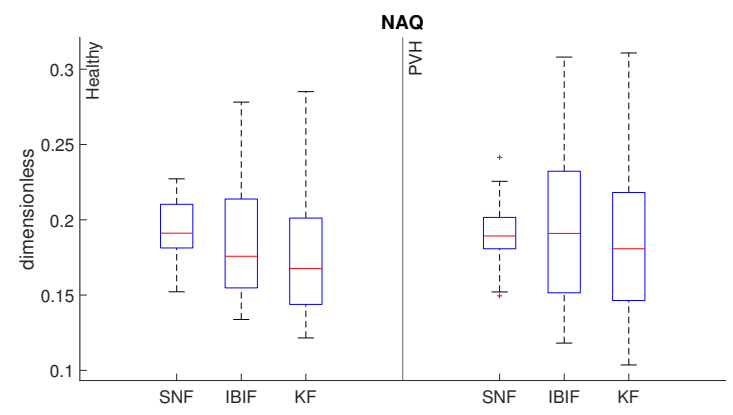

(d)

Figure 10. Distribution of average measures from the Rainbow passage for 50 vocally-healthy subjects and 50 PVH subjects. 
/a/ vowel, which in some cases could affect estimated glottal features up to $50 \%$ in error [61].

Figure 12 shows a voiced segment from a PVH female subject. In this case, the IBIF methods overestimates the peak-to-peak values from the SNF method (RMSE = 229.4. $\mathrm{ml} / \mathrm{s}$ ). Also, the opening phase is faster compared to ground truth. The KF method compensates the large amplitude of the IBIF output waveform, while at the same time it gets closer to the ground truth signal in the opening and close phase $(R M S E=76.3 \mathrm{ml} / \mathrm{s})$. As previously stated the IBIF and, therefore, the model used by the Kalman filter, are both calibrated using a procedure based on fitting the vowel /a/. In these cases, the method based on Kalman follows the reference signal a bit closer than IBIF. Even though the Kalman filter is an alternative implementation of the IBIF filter, the adaptive filtering nature of Kalman allows to track better the ground truth signal than IBIF. Similar trends were found in different subjects and tokens.

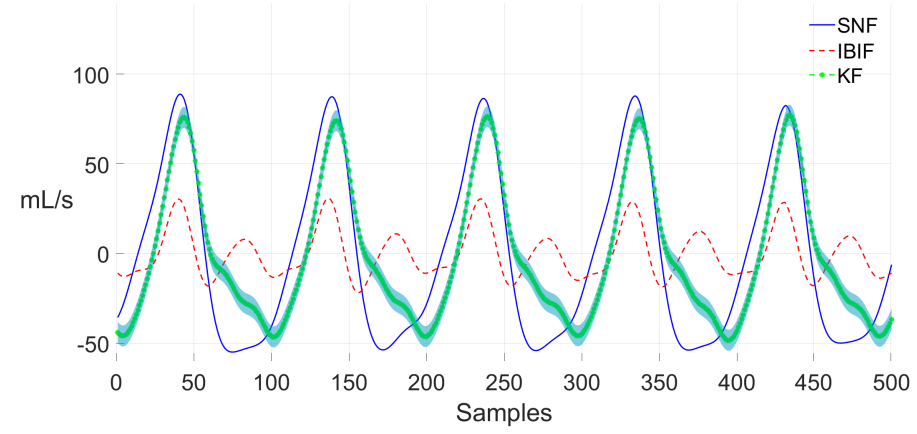

Figure 11. Section from the Rainbow passage (healthy female) with estimations of GVV: Kalman filter (solid and dot green), IBIF (dashed red), and single notch filter (solid blue). The estimation of GVV using Kalman filter includes $\pm 2 \sigma$ (standard deviation) on the green shaded region.

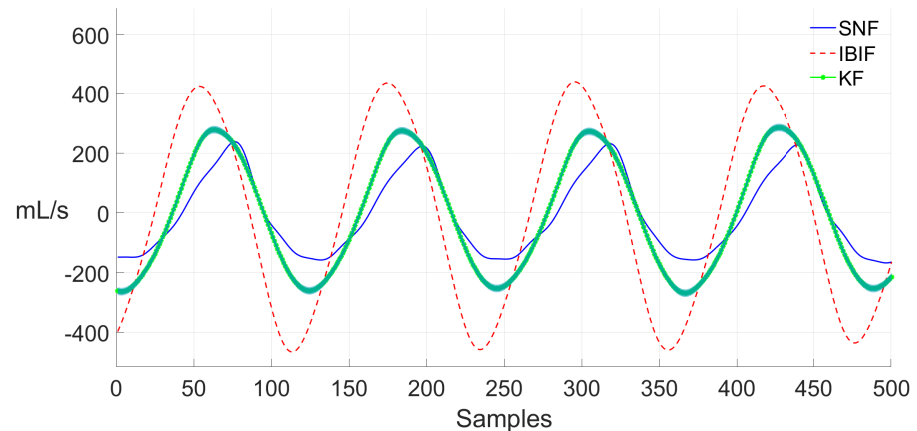

Figure 12. Section from the Rainbow passage (female PVH) with estimations of GVV: Kalman filter (solid and dot green), IBIF (dashed red), and single notch filter (solid blue). The estimation of GVV using Kalman filter includes $\pm 2 \sigma$ (standard deviation) on the green shaded region.

\section{Discussion}

The proposed method based on the modified Kalman filter algorithm is an adaptive implementation of the IBIF scheme, and therefore has some differences with the original IBIF design, namely a forward prediction of the accelerometer signal (i.e., no filter is inverted) and a truncation of the finite impulse response required to reduce the computational burden. In spite of these differences, it is shown that the Kalman filter implementation allows for enhancing the glottal airflow estimates, as it optimally adapts to better predict the accelerometer signal, thus resulting in a closer estimation of the glottal airflow from a Rothenberg mask in benchmark experiments. It is important to note that there are still differences between the Kalman filter glottal airflow estimates and the reference signal from the Rothenberg mask, due to supraglottal inverse filtering errors and measurement 
uncertainty of the oral airflow signal [62]. The small, but significant, differences between the mean values of ACFL and MFDR from PVH subjects can be observed using the IBIF and Kalman method, respectively. These are difficult to assess, particularly, for high-pitched female pathological voices [63]. For example, the method of close phase covariance requires several samples in the closed phase of the glottal flow, which are difficult to obtain for high-pitched subjects [64].

The signal deviations between the Kalman filter and the original (time invariant) FIR IBIF glottal airflow estimates are relatively small, although the former better estimates the amplitude, or peak-to-peak flow, compared to IBIF. These differences can be relevant in some cases, depending on the application. When assessing the relevance of these differences in the context of a classification task to discriminate between vocal fold nodules patients and control subjects using ambulatory accelerometer data, no significant variations in the classification were found, even when comparing frames with low and high error (or deviation) [65]. Thus, the classification task seems to be fairly insensitive to the uncertainty of the airflow estimates from IBIF model parameters, sensor positioning, and other effects. This supports the use of the original FIR version of the IBIF scheme for such classification tasks, which indicates that factors affecting the classification performance in [27] were not degraded by the airflow estimates. However, other applications more sensitive to signal quality (for instance, the estimation of glottal biomechanics and assessment of tissueflow-acoustic interaction [66]) can further benefit from the enhancement offered by the proposed Kalman implementation to estimate more accurate glottal airflow in running speech and/or ambulatory scenarios.

The main differences between the Kalman filter and SNF approach can be observed in $\mathrm{H} 1-\mathrm{H} 2$ and NAQ measures, which are related to low-frequency content and closed quotient, respectively. The IBIF method shows similar differences as well. In order to better estimate these measures, it is necessary to correctly detect the upward and downward slope of the glottal cycle, as well as the closed phase portion. Undue modelled rapid changes in the signal trajectory might induce errors in the Kalman approach which affect the detection of those landmarks in the glottal cycle. In addition, for some subjects, errors in the parameters from IBIF due to calibration could carry through to the Kalman implementation since the latter is built upon the IBIF scheme.

The main current limitation of the proposed Kalman filter approach is the relative high computational cost due to the FIR model used, which can become a problem when processing many hours of recordings (as in ambulatory monitoring) in numerous subjects. Future efforts can be devoted to optimize the approach via more efficient methods, using for example an autoregressive model in the construction of the state space model. Other variations in the construction, e.g., addition of a random walk term or an extended Kalman filter could be investigated as well to encompass non-linear implementations of the accelerometer signal to glottal airflow signal transformation. Flow estimation can also be improved by considering the backward Kalman smoother algorithm, at the expense of an increase in the computational burden and the memory requirements. New model strategies suitable for Kalman filter and smoother would be explored in the future [67].

\section{Conclusions}

A Kalman filter implementation of the subglottal impedance-based inverse filtering scheme was introduced to enhance the estimated glottal airflow from recordings of a neck-surface vibration signal and to assess the relevance of model uncertainty in such estimates. The proposed approach is capable of adapting the signal estimates to correct for inverse filtering deviations, as observed in benchmark experiments with different sustained vowels. Future work is related to the exploration of other applications that can further benefit from the Kalman filter enhancement when estimating glottal airflow and to reduce its computational expense.

Author Contributions: Conceptualization, J.P.C.; data curation, J.P.C. and V.M.E.; formal analysis, J.P.C. and M.Z.; funding acquisition, J.P.C., M.Z., and R.E.H.; investigation, J.P.C., G.A.A., M.Z., J.I.Y., 
A.J.W., V.M.E.; methodology, J.P.C., G.A.A., M.Z., J.I.Y., and A.J.W.; project administration, M.Z.; experimental resources, D.D.M. and R.E.H.; supervision, M.Z.; writing—original draft preparation, J.P.C. and M.Z.; writing-review and editing, M.Z., G.A.A., M.Z., Y.I.Y., A.J.W, V.M.E., D.D.M. and R.E.H. All authors have read and agreed to the published version of the manuscript.

Funding: This research was funded by ANID grants FONDECYT 1191369, BASAL FB0008, (awarded to Matías Zanãrtu), Programa de Incentivos a la Iniciación Científica (PIIC) from Universidad Santa María (awarded to Juan P. Cortés), and the National Institutes of Health (NIH) National Institute on Deafness and Other Communication Disorders of the National Institutes of Health under award numbers P50DC015446 and R33DC011588 (awarded to Robert E. Hillman). The content is solely the responsibility of the authors and does not necessarily represent the official views of the NIH.

Institutional Review Board Statement: The study was conducted according to the guidelines of the Declaration of Helsinki, and approved by the Institutional Review Board (or Ethics Committee) of Mass General Brigham (protocol code 2011P002376, approval date: 8/25/2021).

Informed Consent Statement: Informed consent was obtained from all subjects involved in the study.

Data Availability Statement: Partners Healthcare and MGH are not allowed to give access to data without the Principal Investigator(PI) for the human studies protocol first submitting a protocol amendment to request permission to share the data with a specific collaborator on a case-by-case basis. This policy is based on very strict rules dealing with the protection of patient data and information. Anyone wishing to request access to the data must first contact Ms. Sarah Derosa, Program Coordinator for Research and Clinical Speech-Language Pathology, Center for Laryngeal Surgery and Voice Rehabilitation, Massachusetts General Hospital: sederosa@partners.org

Conflicts of Interest: Dr. Matías Zañartu has a financial interest in Lanek SPA, a company focused on developing and commercializing biomedical devices and technologies. Dr. Zañartu's interests were reviewed and are managed by Universidad Técnica Federico Santa María in accordance with its conflict-of-interest-policies. Drs. Robert Hillman and Daryush Mehta have a financial interest in InnoVoyce LLC, a company focused on developing and commercializing technologies for the prevention, diagnosis, and treatment of voice-related disorders. Dr. Hillman's and Dr. Mehta's interests were reviewed and are managed by Massachusetts General Hospital and Mass General Brigham in accordance with their conflict-of-interest policies. The funders had no role in the design of the study; in the collection, analyses, or interpretation of data; in the writing of the manuscript, or in the decision to publish the results.

1. Bhattacharyya, N. The prevalence of voice problems among adults in the United States. The Laryngoscope 2014, 124, 2359-2362. doi:10.1002/lary.24740.

2. Coyle, S.M.; Weinrich, B.D.; Stemple, J.C. Shifts in Relative Prevalence of Laryngeal Pathology in a Treatment-Seeking Population. Journal of Voice 2001, 15, 424-440. doi: 10.1016/S08921997(01)00043-1, doi:10.1016/S0892-1997(01)00043-1.

3. Herrington-Hall, B.; Lee, L.; Stemple, J.C.; Niemi, K.R.; McHone, M.M. Description of laryngeal pathologies by age, sex, and occupation in a treatment-seeking sample. Journal of Speech and Hearing Disorders 1988, 53, 57-64. doi:10.1044/jshd.5301.57.

4. Kridgen, S.; Hillman, R.E.; Stadelman-Cohen, T.; Zeitels, S.; Burns, J.A.; Hron, T.; Krusemark, C.; Muise, J.; Stan, J.H.V. Patient-Reported Factors Associated with the Onset of Hyperfunctional Voice Disorders. Annals of Otology, Rhinology and Laryngology 2021, 130, 389-394. doi: 10.1177/0003489420956379.

5. Hillman, R.E.; Holmberg, E.B.; Perkell, J.S.; Walsh, M.; Vaughan, C. Objective Assessment of Vocal Hyperfunction: An Experimental Framework and Initial Results. J Speech Hear. Res. 1989, 32, 373-392.

6. Mehta, D.D.; Hillman, R.E. Use of Aerodynamic Measures in Clinical Voice Assessment. Voice and Voice Disorders 2007, 17, 14-18.

7. Hillman, R.E.; Stepp, C.E.; Stan, J.H.V.; Zañartu, M.; Mehta, D.D. An Updated Theoretical Framework for Vocal Hyperfunction. American Journal of Speech-Language Pathology 2020, 29, 2254-2260, [https://pubs.asha.org/doi/pdf/10.1044/2020 A JSLP - 20 - 00104]. doi: 10.1044/2020\_AJSLP-20-00104. 
8. Franco, R.A.; Andrus, J.G. Common Diagnoses and Treatments in Professional Voice Users. Otolaryngologic Clinics of North America 2007, 40, 1025-1061. doi:10.1016/j.otc.2007.05.008.

9. Espinoza, V.M.; Zañartu, M.; Van Stan, J.H.; Mehta, D.D.; Hillman, R.E. Glottal Aerodynamic Measures in Women With Phonotraumatic and Nonphonotraumatic Vocal Hyperfunction. Journal of Speech, Language, and Hearing Research 2017, 60, 2159-2169.

10. Holmberg, E.B.; Doyle, P.; Perkell, J.S.; Hammarberg, B.; Hillman, R.E. Aerodynamic and Acoustic Voice Measurements of Patients with Vocal Nodules: Variation in Baseline and Changes Across Voice Therapy. J. Voice 2003, 17, 269-282.

11. Lien, Y.A.S.; Calabrese, C.R.; Michener, C.M.; Murray, E.H.; Van Stan, J.H.; Mehta, D.D.; Hillman, R.E.; Noordzij, J.P.; Stepp, C.E. Voice Relative Fundamental Frequency Via Neck-Skin Acceleration in Individuals With Voice Disorders. Journal of Speech, Language, and Hearing Research 2015, 58, 1482-1487.

12. Mehta, D.D.; Espinoza, V.M.; Van Stan, J.H.; Zañartu, M.; Hillman, R.E. The difference between first and second harmonic amplitudes correlates between glottal airflow and neck-surface accelerometer signals during phonation. The Journal of the Acoustical Society of America 2019. accepted for publication.

13. Narasimhan, S.V.; Vishal, K. Spectral Measures of Hoarseness in Persons with Hyperfunctional Voice Disorder. J. Voice 2016, 31, 57 - 61.

14. Mehta, D.D.; Van Stan, J.H.; Zañartu, M.; Ghassemi, M.; Guttag, J.V.; Espinoza, V.M.; Cortés, J.P.; Cheyne, H.A.I.; Hillman, R.E. Using ambulatory voice monitoring to investigate common voice disorders: research update. Front. Bioeng. Biotechnol. 3:155. doi: 10.3389/fbioe.2015.00155 2015.

15. Stan, J.H.V.; Mehta, D.D.; Ortiz, A.J.; Burns, J.A.; Toles, L.E.; Marks, K.L.; Vangel, M.; Hron, T.; Zeitels, S.; Hillman, R.E. Differences in Weeklong Ambulatory Vocal Behavior Between Female Patients With Phonotraumatic Lesions and Matched Controls. Journal of speech, language, and hearing research : JSLHR 2020, 63, 372-384. doi:10.1044/2019_JSLHR-19-00065.

16. Stan, J.H.V.; Mehta, D.D.; Ortiz, A.J.; Burns, J.A.; Marks, K.L.; Toles, L.E.; Stadelman-Cohen, T.; Krusemark, C.; Muise, J.; Hron, T.; Zeitels, S.M.; Fox, A.B.; Hillman, R.E. Changes in a Daily Phonotrauma Index After Laryngeal Surgery and Voice Therapy: Implications for the Role of Daily Voice Use in the Etiology and Pathophysiology of Phonotraumatic Vocal Hyperfunction. Journal of Speech, Language, and Hearing Research 2020, pp. 1-11. doi: 10.1044/2020_JSLHR-20-00168.

17. Stan, J.H.V.; Ortiz, A.J.; Cortes, J.P.; Marks, K.L.; Toles, L.E.; Mehta, D.D.; Burns, J.A.; Hron, T.; Stadelman-Cohen, T.; Krusemark, C.; Muise, J.; Fox-Galalis, A.B.; Nudelman, C.; Zeitels, S.; Hillman, R.E. Differences in Daily Voice Use Measures Between Female Patients With Nonphonotraumatic Vocal Hyperfunction and Matched Controls. JSLHR Research Article Journal of Speech, Language, and Hearing Research • 2021, 64, 1457-1470. doi:10.23641/asha.

18. Manfredi, C.; Bruschi, T.; Dallai, A.; Ferri, A.; Tortoli, P.; Calisti, M. Voice quality monitoring: A portable device prototype. 2008 30th Annual International Conference of the IEEE Engineering in Medicine and Biology Society, 2008, pp. 997-1000. doi:10.1109/IEMBS.2008.4649323.

19. Smith, N.R.; Rivera, L.A.; Dietrich, M.; Shyu, C.R.; Page, M.P.; DeSouza, G.N. Detection of Simulated Vocal Dysfunctions Using Complex sEMG Patterns. IEEE Journal of Biomedical and Health Informatics 2016, 20, 787-801. doi:10.1109/JBHI.2015.2490087.

20. Cheyne, H.A.; Hanson, H.M.; Genereux, R.P.; Stevens, K.N.; Hillman, R.H. Development and Testing of a Portable Vocal Accumulator. J Speech Hear. Res. 2003, 46, 1457-1467.

21. Carroll, T.; Nix, J.; Hunter, E.; Titze, I.; Abaza, M. Objective Measurement of Vocal Fatigue in Classically Trained Singers: A Pilot Study of Vocal Dosimetry Data. Otolarynol. Head Neck Surg. 2006, 135, 595-602.

22. Titze, I.R.; Hunter, E.J.; Svec, J.G. Voicing and Silence Periods in Daily and Weekly Vocalizations of Teachers. J. Acoust. Soc. Am. 2007, 121, 469-478.

23. Mehta, D.D.; Zañartu, M.; Feng, S.W.; Cheyne, H.A.; Hillman, R.E. Mobile Voice Health Monitoring Using a Wearable Accelerometer Sensor and a Smartphone Platform. Biomedical Engineering, IEEE Transactions on 2012, 59, 3090-3096. doi:10.1109/TBME.2012.2207896.

24. Ghassemi, M.; Van Stan, J.; Mehta, D.; Zañartu, M.; Cheyne, H.; Hillman, R.; Guttag, J. Learning to Detect Vocal Hyperfunction From Ambulatory Neck-Surface Acceleration Features: Initial Results for Vocal Fold Nodules. Biomedical Engineering, IEEE Transactions on 2014, 61, 16681675. doi:10.1109/TBME.2013.2297372.

25. Van Stan, J.H.; Mehta, D.D.; Zeitels, S.M.; Burns, J.A.; Barbu, A.M.; Hillman, R.E. Average Ambulatory Measures of Sound Pressure Level, Fundamental Frequency, and Vocal Dose Do 
Not Differ Between Adult Females With Phonotraumatic Lesions and Matched Control Subjects. Annal. Otolog. Rhinol. Laryngol. 2015, 124, 864-874.

26. Bottalico, P.; Graetzer, S.; Astolfi, A.; Hunter, E. Silence and Voicing Accumulations in Italian Primary School Teachers With and Without Voice Disorders. Journal of Voice 2017, 31. doi: 10.1016/j.jvoice.2016.05.009.

27. Cortés, J.P.; Espinoza, V.M.; Ghassemi, M.; Mehta, D.D.; Van Stan, J.H.; Hillman, R.E.; Guttag, J.V.; Zañartu, M. Ambulatory assessment of phonotraumatic vocal hyperfunction using glottal airflow measures estimated from neck-surface acceleration. PLOS ONE 2018, 13, 1-22. doi: 10.1371/journal.pone.0209017.

28. Espinoza, V.M.; Mehta, D.D.; Stan, J.H.V.; Hillman, R.E.; Zañartu, M. Glottal aerodynamics estimated from neck-surface vibration in women with phonotraumatic and nonphonotraumatic vocal hyperfunction. Journal of Speech, Language, and Hearing Research 2020, 63, 2861-2869. doi:10.1044/2020_JSLHR-20-00189.

29. Zañartu, M.; Galindo, G.; Erath, B.D.; Peterson, S.D.; Wodicka, G.R.; Hillman, R.E. Modeling the effects of a posterior glottal opening on vocal fold dynamics with implications for vocal hyperfunction. J. Acoust. Soc. Am. 2014, 136, 3262-3271.

30. Galindo, G.E.; Peterson, S.D.; Erath, B.D.; Castro, C.; Hillman, R.E.; Zañartu, M. Modeling the pathophysiology of phonotraumatic vocal hyperfunction with a triangular glottal model of the vocal folds. Journal of Speech, Language, and Hearing Research 2017, 60, 2452-2471.

31. Rothenberg, M. A new inverse filtering technique for deriving the glottal air flow waveform during voicing. The Journal of the Acoustical Society of America 1973, 53, 1632-1645. doi: http:/ /dx.doi.org/10.1121/1.1913513.

32. Zañartu, M.; Ho, J.C.; Mehta, D.D.; Hillman, R.E.; Wodicka, G.R. Subglottal ImpedanceBased Inverse Filtering of Voiced Sounds Using Neck Surface Acceleration. Audio, Speech, and Language Processing, IEEE Transactions on 2013, 21, 1929-1939. doi: 10.1109/TASL.2013.2263138.

33. Espinoza, V. Stationary And Dynamic Aerodynamic Assessment Of Vocal Hyperfunction Using Enhanced Supraglottal And Subglottal Inverse Filtering Methods. PhD thesis, Universidad Técnica Federico Santa María, Valparaíso, Chile, 2018.

34. Perkell, J.S.; Holmberg, E.B.; Hillman, R.E. A system for signal processing and data extraction from aerodynamic, acoustic, and electroglottographic signals in the study of voice production. The Journal of the Acoustical Society of America 1991, 89, 1777-1781. doi: http:/ /dx.doi.org/10.1121/1.401011.

35. Stevens, K.N. Acoustic Phonetics; MIT Press, 2000.

36. Ho, J.C.; Zañartu, M.; Wodicka, G.R. An Anatomically Based, Time-Domain Acoustic Model of the Subglottal System for Speech Production. J. Acoust. Soc. Am. 2011, 129, 1531-1547.

37. Zañartu, M.; Espinoza, V.M.; Mehta, D.D.; Van Stan, J.H.; Cheyne III, H.A.; Ghassemi, M.; Guttag, J.V.; Hillman, R.E. Toward An Objective Aerodynamic Assessment Of Vocal Hyperfunction Using A Voice Health Monitor. 8th International Workshop on Models and Analysis of Vocal Emissions for Biomedical Applications, MAVEBA 2013, December 16 - 18 2013, Firenze, Italy., 2013.

38. Espinoza, V.M.; Mehta, D.D.; Van Stan, J.H.; Hillman, R.E.; Zañartu, M. Uncertainty of glottal airflow estimation during continuous speech using impedance-based inverse filtering of the neck-surface acceleration signal. Proceedings of the Acoustical Society of America 2017.

39. Arulampalam, M.S.; Maskell, S.; Gordon, N.; Clapp, T. A tutorial on particle filters for online nonlinear/non-Gaussian Bayesian tracking. IEEE Transactions on Signal Processing 2002, 50, 174-188.

40. Benesty, J.; Sondhi, M.; Huang, Y. Springer Handbook of Speech Processing; Springer, 2008.

41. Fant, G. The Acoustic Theory of Speech Production; Mouton \& Co. N.V. Publishers, 1960.

42. Zañartu, M. Acoustic Coupling in Phonation and its Effect on Inverse Filtering of Oral Airflow and Neck Surface Acceleration. PhD thesis, Purdue University, West Lafayette, IN, 2010.

43. Rosenberg, A.E. Effect of Glottal Pulse Shape on the Quality of Natural Vowels. The Journal of the Acoustical Society of America 1971, 49, 583-590.

44. Fant, G.; Liljencrants, J.; Lin, Q. A Four-Parameter Model of Glottal Flow. STL-QPSR 1985, 4.

45. Alzamendi, G.; Schlotthauer, G. Modeling and joint estimation of glottal source and vocal tract filter by state-space methods. Biomedical Signal Processing and Control 2017, 37.

46. Doval, B.; D'Alessandro, C.; Henrich Bernardoni, N. The spectrum of glottal flow models. Acta Acustica united with Acustica 2006, 92, 1026-1046. 
47. Markel, J.; Gray, A. Linear Prediction of Speech; Communication and cybernetics, SpringerVerlag, 1976.

48. Proakis, J.G.; Manolakis, D.G. Digital Signal Processing: Principles, Algorithms and Applications, 4th ed.; Pearson Education Inc., 2007.

49. Oppenheim, A.V.; Schafer, R.; Buck, J. Discrete-Time Signal Processing; Prentice Hall: Englewood Cliffs, N.J., 1999.

50. Rabiner, L.R.; Schafer, R.W. Theory and Applications of Digital Speech Processing; Prentice Hall, 2010.

51. Grewal, M.; Andrews, A. Kalman Filtering: Theory and Practice Using MATLAB; Wiley, 2008.

52. Kunduk, M.; Mcwhorter, A. True vocal fold nodules: The role of differential diagnosis. Current opinion in otolaryngology \& head and neck surgery 2009, 17, 449-52.

53. Alisa, Z.; Danielle, B.; M., K.S.; Thomas, M.; Lucian, S. Gender and age in benign vocal fold lesions. The Laryngoscope, 125, 191-196. doi:10.1002/lary.24911.

54. Mehta, D.D.; Hillman, R.E. Voice Assessment: Updates on Perceptual, Acoustic, Aerodynamic, and Endoscopic Imaging Methods. Curr. Opin. Otolaryngol. Head Neck Surg. 2008, 16, $211-$ 215.

55. Hogikyan, N.D.; Sethuraman, G. Validation of an instrument to measure voice-related quality of life (V-RQOL). Journal of Voice 1999, 13, 557-569. doi: 10.1016/S0892-1997(99)80010-1, doi: 10.1016/S0892-1997(99)80010-1.

56. Kempster, G.B.; Gerratt, B.R.; Abbott, K.V.; Barkmeier-Kraemer, J.; Hillman., R.E. Consensus Auditory-Perceptual Evaluation of Voice: Development of a Standardized Clinical Protocol. American Journal of Speech-Languaje Pathology 2009, 18, 124-132.

57. Fairbanks, G. Voice and Articulation Drillbook; NY: Harper and Row, 1960.

58. Holmberg, E.B.; Hillman, R.E.; Perkell, J.S. Glottal Air-Flow and Transglottal Air-Pressure Measurements for Male and Female Speakers in Soft, Normal, and Loud Voice. J. Acoust. Soc. Am. 1988, 84, 511-529.

59. $\overline{\mathrm{Alku}}$, P.; Bäckström, T.; Vilkman, E. Normalized amplitude quotient for parametrization of the glottal flow. The Journal of the Acoustical Society of America 2002, 112, 701-710. doi: http:/ /dx.doi.org/10.1121/1.1490365.

60. Brown, M.B.; Forsythe, A.B. Robust Tests for the Equality of Variances. Journal of the American Statistical Association 1974, 69, 364-367, [https: / / www.tandfonline.com/doi/pdf/10.1080/01621459.197 doi:10.1080/01621459.1974.10482955.

61. Manriquez, R.; Espinoza, V.; Castro, C.; Cortes, J.; Zañartu, M. Parameter analysis and uncertainties of impedance-based inverse filtering from neck surface acceleration. 14th International Conference on Advances in Quantitative Laryngology, Voice and Speech Research (AQL 2021); , 2021.

62. May, N.A.; Scherer, R.C. Airflow Error Measurement Due to Pneumotachograph Mask Rim Leaks. Journal of Voice 2018, 32, 403-419. doi:https:/ / doi.org/10.1016/j.jvoice.2017.07.016.

63. Drugman, T.; Alku, P.; Alwan, A.; Yegnanarayana, Y. Glottal source processing: From analysis to applications. Computer Speech \& Language 2014, 28, 1117-1138.

64. Alku, P.; Magi, C.; Yrttiaho, S.and Bäckström, T.; Story, B. Closed phase covariance analysis based on constrained linear prediction for glottal inverse filtering. J. Acoust. Soc. Am. 2009, 125, 3289-3305.

65. Cortés, J.; Alzamendi, G.; Weinstein, A.; Yuz, J.; Espinoza, V.; Mehta, D.; Van Stan, J.; Hillman, R.; Zañartu, M. Uncertainty of ambulatory airflow estimates and its effect on the classification of phonotraumatic vocal hyperfunction. 13th International Conference on Advances in Quantitative Laryngology, Voice and Speech Research (AQL 2019); , 2019.

66. Ibarra, E.J.; Parra, J.A.; Alzamendi, G.A.; Cortés, J.P.; Espinoza, V.M.; Mehta, D.D.; Hillman, R.E.; Zañartu, M. Estimation of Subglottal Pressure, Vocal Fold Collision Pressure, and Intrinsic Laryngeal Muscle Activation From Neck-Surface Vibration Using a Neural Network Framework and a Voice Production Model. Frontiers in Physiology 2021, 12, 1419 . doi: 10.3389/fphys.2021.732244.

67. Morales, A.; Yuz, J.I. Reduced order modeling for glottal airflow estimation using a Kalman smoother. 2021 IEEE International Conference on Automation/XXIV Congress of the Chilean Association of Automatic Control (ICA-ACCA), 2021, pp. 1-6. doi: 10.1109/ICAACCA51523.2021.9465282. 
